\title{
Uma Revisão dos Artigos Publicados no Journal of Applied Behavior Analysis (JABA) sobre Comportamento Verbal e Autismo entre 2008 e 2012
}

Un Revisión de los Artículos Publicados en Journal of Applied Behavior Analysis (JABA) sobre Comportamiento Verbal y Autismo entre 2008 y 2012

A Review of Articles Published in Journal of Applied Behavior Analysis
(JABA) on Verbal Behavior and Autism between 2008 and 2012 Maria Carolina Côrrea Martone ${ }^{1}$ e Larissa Helena Zani Santos-Carvalho²

[1] Universidade Federal de São Carlos e Núcleo Paradigma de Análise do Comportamento [2] Universidade Federal de São Carlos | Título abreviado: Revisão de Artigos do JABA sobre Comportamento Verbal e Autismo I Endereço para correspondência: Rua Wanderley, 611. CEP: 05011-001. São Paulo, SP. Email: carolina.martone@uol.com.br

Resumo: O presente estudo faz uma breve apresentação sobre o transtorno do espectro autista (TEA), bem como sobre as categorias gerais de trabalho referentes ao ensino de linguagem para alunos da educação especial na análise aplicada do comportamento. O objetivo foi realizar uma revisão dos artigos publicados no Journal of Applied Behavior Analysis (JABA) entre 2008 e 2012. A revisão foi realizada por meio da base de dados PubMed e o critério de seleção dos artigos incluiu as palavras comportamento verbal e autismo no corpo do texto. Vinte e sete artigos foram selecionados e analisados por (a) ano da publicação, (b) filiação dos autores, (c) número de participantes de cada estudo, (d) tema, (e) procedimentos utilizados e (f) tipo de artigo. As revisões foram excluídas. Os resultados mostraram que o ensino de mando foi o tema mais estudado e que controle de estímulos e reforçamento positivo foram os procedimentos mais frequentes. Além disso, universidades e centros prestadores de serviço têm publicado juntos, o que mostra que o desenvolvimento das estratégias de ensino tem se dado de forma integrada com profissionais que atuam na área e lidam com questões práticas referentes às dificuldades dessa população.

Palavras-chave: comportamento verbal, autismo, revisão, Journal of Applied Behavior Analysis.

Abstract: This article makes a brief presentation on autism spectrum disorder (ASD) as well as on general categories of studies related to language teaching to learners of special education in applied behavior analysis. The aim of this study was to conduct a review of articles published in the Journal of Applied Behavior Analysis (JABA) between 2008 and 2012. The articles were selected from the PubMed database and the criteria for selection included the words verbal behavior and autism. Twenty-seven articles were selected and analyzed according to (a) year of publication, (b) affiliation of authors, (c) number of participants, (d) subject, (e) procedures and (f) type of article. The reviews were left out. The results showed that the mand teaching was the most studied theme and that stimulus control and positive reinforcement 
were the procedures employed with frequency. Moreover, it was observed that universities and service providers have published studies together, which shows that the development of teaching strategies has occurred in an integrated basis with professional work in the area and deals with practical issues related to the difficulties of this population.

Key words: verbal behavior, autism, review, Journal of Applied Behavior Analysis.

Resumen: En este estudio se hace una presentación de las características del trastorno del espectro autista, así como una breve descripción de la metodología en dos principales corrientes para la enseñanza del idioma a las personas con autismo dentro del área de analisis aplicado del comportamiento. El objetivo de este estudio fue realizar una revisión de los artículos publicados en Journal of Applied Behavior Analysis (JABA) entre 2008 y 2012. La revisión se llevó a cabo a través de PubMed base de datos y los criterios para la selección de los artículos incluidos el autismo palabras en el comportamiento verbal y el texto del cuerpo. Veinte y siete artículos fueron selecionados y analisados de acuerdo con (a) año de publicación, (b) la afiliación de los autores, (c) número de participantes, (d) la tematica y (e) los procedimentos. Las revisiones han sido excluidas. Los resultados mostraron que la enseñanza de mando ha sido la temática más estudiada y que el control de estímulos y reforzamiento positivo han sido los procedimientos más frecuentes. Además, universidades y centros que prestan servicios fueron publicados juntos, lo que muestra que el desarrollo de estrategias de enseñanza ha ocurrido de forma integrada con profesionales que actúan en el área y se ocupan con cuestiones prácticas referentes a las dificultades de esta populación.

Palabras-clave: conducta verbal, autismo, revisión, Journal of Applied Behavior Analysis. 
A palavra autismo foi utilizada pela primeira vez em 1911, pelo psiquiatra Eugene Bleuler, e descrevia um dos sintomas observados na esquizofrenia. Contudo, o autismo, como um quadro diferenciado de outros, foi caracterizado em 1943 pelo médico austríaco Leo Kanner. O nome foi proposto para chamar a atenção para o prejuízo severo na interação social que era muito evidente desde o início da vida desses pacientes. As características descritas pelo autor incluíam desinteresse pelo mundo ao redor, boa capacidade de memorização mecânica, mutismo ou linguagem sem intenção comunicativa efetiva, hipersensibilidade aos estímulos e fala repetitiva. Quase na mesma época, em 1944, outro austríaco, Hans Asperger, publicou um estudo no qual descreveu um grupo de crianças com as características observadas por Kanner. Entretanto, apesar das características semelhantes, seu estudo apontou para um grupo de sujeitos com níveis de inteligência e linguagem superiores aos relatados pelo colega. Os achados de Hans Asperger só foram resgatados na década de 1970 e deram origem ao quadro hoje conhecido como síndrome de Asperger (Belsário Filho \& Cunha, 2010).

Com o passar do tempo e o maior conhecimento a respeito desse tipo de condição, outras classificações surgiram. Recentemente, cunhou-se o termo transtorno do espectro autista (TEA) para caracterizar um conjunto muito heterogêneo de alterações comportamentais com início precoce, curso crônico e impacto variável em áreas múltiplas do desenvolvimento (APA, 2013).

Dados atuais americanos apontam para uma estimativa de que $1 \%$ da população infantil apresenta TEA (Rice, 2009). No Brasil, o único estudo de prevalência sugere que o autismo e suas variações afetam uma em cada 370 crianças, ou 0,3\% dessa população, devendo existir aproximadamente 40 mil crianças e adolescentes com TEA no Estado de São Paulo (Paula, Ribeiro, Fombone \& Mercadante, 2011). Para Fombonne (2009), os dados mundiais indicam que o TEA tornou-se um dos transtornos do neurodesenvolvimento mais comuns, com um alto impacto pessoal, familiar e social. Apesar de ser difícil avaliar custos humanos e sociais para criança com TEA e para seus familiares, dados provenientes de países desenvolvidos revelam, por exemplo, que crianças com TEA frequentam nove vezes mais os serviços de saúde do que crianças com outros problemas médicos e três vezes mais que crianças com deficiência mental (Newschaffer et al., 2007). A implementação de intervenções precoces, estruturadas e prolongadas propicia um melhor prognóstico e uma redução de custos financeiros e sociais para as famílias e os sistemas públicos de educação e saúde (Werner, Dawson, Munson \& Osterling, 2005).

As manifestações comportamentais que definem o transtorno incluem déficits qualitativos na interação social e na comunicação, padrões de comportamento repetitivos e estereotipados e um repertório restrito de interesses e atividades (Rapin, 1991).

O comprometimento na sociabilidade pode ser observado, por exemplo, na dificuldade em manter ou sustentar o contato ocular, no compartilhamento de brinquedos e interesses e na ausência de brincadeiras imaginativas com os pares (APA, 2003). Entre as dificuldades de comunicação, observa-se atraso na aquisição das primeiras palavras e frases, uso do corpo de outra pessoa como forma de se comunicar, uso de ecolalias e neologismos, inversão de pronomes e dificuldade em iniciar e/ou manter uma conversa com outra pessoa (APA, 2003).

Quanto aos interesses restritos, geralmente referem-se a um interesse de intensidade incomum na sua qualidade, o qual não é desenvolvido dentro de um contexto mais amplo de conhecimento. De forma complementar, os comportamentos estereotipados são observados por maneirismos de mãos e dedos, uso repetitivo de objetos ou interesse em partes específicas do mesmo, sequências fixas de comportamentos e interesses sensoriais incomuns (APA, 2003).

Entre os sinais para detecção de risco para o TEA antes dos 24 meses, destacamos:

- não balbuciar (e.g., "mamã", "baba” e "papá”), - ausência de brincadeiras que envolvam imitação,

- não olhar quando chamado pelo nome,

- não parecer reconhecer pessoas familiares,

- não olhar para onde as pessoas próximas apontam,

- não saber para que servem coisas que já conhece,

- não seguir instruções simples (e.g., "Pegue o brinquedo"), 
- não adquirir novas palavras,

- não perceber ou parecer dar importância quando os pais chegam/saem e

- brincar de forma pouco funcional e repetitiva com brinquedos (Secretaria dos Direitos da Pessoa com Deficiência, 2013).

A combinação dos aspectos observados acima dificulta a participação em brincadeiras, atividades escolares ou outros eventos sociais (Suplino, 2007). Duas falhas de repertório significativas e usualmente frequentes em pessoas com TEA, as quais envolvem comprometimento nas áreas de comunicação e socialização, são a atenção compartilhada e a capacidade de perceber os outros e a si próprio.

Bosa (2001) define atenção compartilhada como a habilidade de envolver a alternância do olhar e outros sinais comunicativos entre um parceiro e o objeto/evento. Para Lampreia (2007), essa habilidade tem sido considerada muito importante nos bebês por ser a precursora da compreensão das intenções comunicativas dos outros, da imitação e da linguagem. Já a formulação teórica que descreve a habilidade de perceber a si e aos outros é conhecida como teoria da mente e, em linhas gerais, descreve a habilidade que os seres humanos têm de atribuir estados mentais (e.g., crenças, intenções e desejos) a si próprio e aos outros (Baron-Cohen, 2000).

Segundo Nunes e Nunes Sobrinhos (2003), os déficits na atenção compartilhada e na teoria da mente condensam aspectos determinantes do distúrbio e fazem com que a aquisição da linguagem se torne primordial nos programas de intervenção precoce. De acordo com a revisão de LeBlanc e Gillis (2012), a implementação de intervenções precoces, psicoeducacionais e prolongadas propicia melhor prognóstico, bem como redução de custos financeiros e sociais para as famílias, sendo que as intervenções com resultados mais efetivos derivam da teoria comportamental.

Nos últimos 50 anos, a análise aplicada do comportamento (AAC) tem demonstrado cientificamente a eficácia de suas propostas com pesquisas e planos de intervenção bem sucedidos (Reichow \& Volkmar, 2010), incluindo indivíduos com TEA. Particularmente para essas pessoas, os ganhos são observáveis devido ao aumento global no repertório comportamental, sobretudo em áreas como as de funcionamento intelectual e social, desenvolvi- mento da linguagem e aquisição de habilidades de vida diária (Virués-Ortega, 2010).

Em geral, crianças com TEA apresentam dois problemas combinados: falhas e excessos no repertório comportamental. Por um lado, a ausência ou escassez de comportamentos relevantes, tais como contato visual e verbalizações espontâneas; por outro, a presença de agressões e estereotipias. A AAC apresenta um conjunto de ferramentas e uma estrutura de trabalho importantes para o ensino e o manejo desses problemas, além de guiar o terapeuta ou professor a maximizar o ensino em uma relação de trabalho individualizada.

Para Carr e Firth (2005), dois marcos foram responsáveis pela divulgação em larga escala dos princípios da análise do comportamento junto às pessoas com TEA: as publicações do artigo de Lovaas (1987) sobre intervenção comportamental intensiva e precoce (Early and Intensive Behavioral Intervention - EIBI) e do livro de Catherine Maurice (1993), que descreveu o tratamento de seus dois filhos autistas por meio da AAC.

O trabalho de Lovaas (1987) foi um estudo de longo prazo sobre tratamento comportamental intensivo para crianças com autismo. O tratamento incluía, em linhas gerais, 40 horas semanais de trabalho direto com a crianças por meio da execução de um currículo estruturado e dividido em áreas do aprendizado (acadêmica, social, lazer e linguagem). Dois grupos de crianças foram observados: grupo intervenção e grupo controle. Os resultados mostraram que no grupo intervenção, de 19 crianças, $47 \%$ das que receberam tratamento comportamental intensivo atingiram níveis normais de funcionamento intelectual e educacional, com coeficiente de inteligência na faixa do normal e uma performance adequada na escola pública. Ainda dessa amostra, $40 \%$ foram posteriormente diagnosticados com retardo leve e apenas $10 \%$ diagnosticados com retardo severo. Comparativamente, no grupo controle (que não recebeu o tratamento proposto por Lovaas), somente $2 \%$ atingiram funcionamento educacional e intelectual normais, $45 \%$ foram diagnosticados com retardo leve e $53 \%$ foram diagnosticados com retardo severo. 
O livro de Catherine Maurice, Let Me Hear Your Voice: A Family Triumph Over Autism ${ }^{1}$, destacou para o público o tratamento do autismo por meio da AAC, incluindo a descrição dos programas de ensino que foram aplicados às crianças. A eficácia da intervenção (os filhos de Maurice foram considerados "recuperados") guiou outros pais a considerar essa forma de tratamento.

A disseminação dos princípios da análise do comportamento em seu viés aplicado fomentou a publicação de diversos manuais e livros descrevendo procedimentos comportamentais e currículos para o ensino de habilidades para crianças autistas (Barbera, 2007; Greer \& Ross, 2008; Leaf, McEachin \& Harsh, 1999; Lovaas, 2003; Maurice, Green \& Luce, 1996), sempre com algum grau de influência oriunda da abordagem curricular proposta por Lovaas e seus colegas da Universidade da Califórnia, em Los Angeles.

O modelo desenvolvido por Lovaas é apontado como a influência mais significativa sobre a metodologia de ensino utilizada por praticantes da análise do comportamento nos últimos anos (Carr \& Firth, 2005). Inclui, em linhas gerais, tratamento intensivo de 30 a 40 horas semanais, elaboração de programas para desenvolver habilidades em diferentes áreas (e.g., comunicação, habilidades sociais, autocuidado e brincar), criação de muitas oportunidades para a criança praticar aquilo que está faltando em seu repertório, ensino de habilidades em pequenos passos que são apresentados da forma mais simples para a mais complexa (chamado de ensino por tentativas discretas) e uso abundante de reforço positivo para que o ambiente de ensino se torne afetivo e motivador.

Um segundo modelo, amplamente utilizado na área aplicada, é chamado de modelo de comportamento verbal (VB). Ele foca o ensino da linguagem e usa a análise de Skinner (1957) de comportamento verbal como ponto central para o delineamento de um programa (Petursdottir \& Carr, 2011). Entre as publicações que seguem esse modelo, destacamos Barbera (2007), Greer e Ross (2008) e Sundberg e Partington (1998).

1 Em português, Quero Escutar Sua Voz: A Vitória de uma Família sobre o Autismo.
Não obstante distintas em diversos pontos, ambas as categorias representam décadas de pesquisa analítico-comportamental em procedimentos de ensino. A abordagem de Lovaas parece ser mais padronizada entre praticantes e clínicas do que o modelo VB. Para Carr e Firth (2005), isso pode estar relacionado ao fato de que, enquanto a abordagem Lovaas foi desenvolvida em um ambiente universitário, a abordagem VB nasceu da prática clínica e da interpretação do trabalho de Skinner sobre comportamento verbal.

Entre as similaridades dos dois modelos, destacamos: ambos (a) enfatizam a importância de um ambiente de ensino cuidadosamente organizado e de formato estruturado, bem como o uso de reforçamento positivo, treino discriminativo e procedimentos de controle de estímulos; (b) afirmam o valor da intervenção precoce, frequente e diária e (c) usam o formato de ensino por tentativas discretas, embora no modelo Lovaas esse procedimento apareça como central na programa de ensino, e, no modelo VB, apareça em conjunto com as estratégias de treino em ambiente natural (NET).

As principais diferenças entre os modelos incluem: ênfase que o modelo VB dá (a) às estratégias NET e (b) ao uso da teoria de Skinner (1957) sobre comportamento verbal no ensino da linguagem. A abordagem VB emprega uma análise funcional da linguagem para ensinar às crianças múltiplas funções da linguagem (e.g., mando, tato e intraverbal) e para ensinar cada função usando as variáveis controladoras finais específicas - diferentemente da abordagem Lovaas, que ensina comportamento verbal sem levar em conta os antecedentes funcionais e suas consequências relevantes (Petursdottir \& Carr, 2011).

Considerando a relevância do tratamento precoce, o aumento exponencial de casos, a eficácia relatada das intervenções por meio da $\mathrm{AAC}$ e a importância da criação permanente de tecnologias para o desenvolvimento da comunicação entre essa população, o objetivo do presente estudo foi realizar uma análise dos trabalhos publicados entre os anos de 2008 e 2012 sobre comportamento verbal e autismo no Journal of Applied Behavior Analysis (JABA). 


\section{Método}

Foram analisados 27 artigos do JABA. O periódico norte-americano foi selecionado por ser a publicação com o maior número de pesquisas comportamentais aplicadas, bem como por ter significativa produção de artigos da área de deficiências do desenvolvimento em geral, incluindo o autismo. $\mathrm{O}$ JABA tem publicação trimestral e é referência em pesquisa aplicada em diversos países, incluindo o Brasil. Esse fato se deve à constância e à qualidade de suas produções. Criado em 1968, tem se dedicado a pesquisar eventos de relevância social, buscando soluções que melhorem a condição humana.

Os artigos, selecionados na base de dados PubMed, entre os anos de 2008 e 2012, tinham de ter no corpo do texto as palavras autismo e comportamento verbal. Revisões foram excluídas. Como indica a Tabela 1, instituições e centros que mais publicaram no período, tema do estudo de cada artigo, procedimentos mais utilizados e número de participantes foram os tópicos analisados e discutidos. Destacamos ainda algumas das sugestões para estudos futuros.

\section{Resultados e Discussão}

A Tabela 1 apresenta os centros, as universidades e os serviços que mais publicaram no período. O número de artigos por instituição não passou de três e foram elas: Utah State University, The New England Center for Children, Northeastern University, Auburn University, Marcus Institute e Emory University School of Medicine. As instituições que publicaram dois artigos foram University of North Texas, Western Michigan University e National University of Ireland. Todas as demais publicaram um artigo no período. Destacamos que em quase todas as publicações encontramos universidades associadas a centros prestadores de serviço. Esse dado parece interessante pois mostra que o desenvolvimento das estratégias, sobretudo de ensino, tem se dado de forma integrada com profissionais que atuam na área e lidam com questões práticas referentes às dificuldades dessa população. Além disso, essa tendência combina com o perfil da revista, de publicar estudos de relevância social.

Em relação ao número de participantes por estudo, dos 27 artigos, 15 tiveram a participação de 3 sujeitos; 5 estudos foram realizados com 4 participantes; 4 estudos com 2 sujeitos e 3 estudos utilizaram apenas 1 participante. A escolha por delineamentos de sujeito-único, diferentemente de outros delineamentos estatísticos e estudos de grupo utilizados em áreas diferentes da psicologia e da medicina, ocorre devido ao entendimento de que as variáveis controladoras devem ser buscadas no ambiente em que o indivíduo se comporta. Para a análise do comportamento, as diferenças entre sujeitos não compromete a análise de dados e cada sujeito é tratado como um indivíduo particular, distinto de qualquer outro (Velasco, Mijares \& Tomanari, 2010).

\section{Publicações sobre Autismo e Comportamento Verbal entre 2008 e 2012}

Tabela 1 - Síntese das Pesquisas sobre Autismo e Comportamento Verbal publicadas no Journal of Applied Behavior Analysis entre 2008 e 2012

\begin{tabular}{|c|c|c|c|c|c|}
\hline Referência & Título & Afiliação & Assunto da pesquisa & Procedimento & $\begin{array}{l}N^{\circ} \text { dePar- } \\
\text { ticipantes }\end{array}$ \\
\hline $\begin{array}{l}\text { 1. Grow et } \\
\text { al.(2008) }\end{array}$ & $\begin{array}{l}\text { Utility of Extinction- } \\
\text { Induced Response } \\
\text { Variability for the } \\
\text { Selection of Mands }\end{array}$ & $\begin{array}{l}\text { Marcus Institute } \\
\text { Emory University } \\
\text { School of Medicine }\end{array}$ & $\begin{array}{l}\text { Seleção e ensino de } \\
\text { mandos durante pro- } \\
\text { cedimento de extinção }\end{array}$ & $\begin{array}{l}\text { Extinção e treino de } \\
\text { comunicação funcional } \\
\text { (FCT) } \\
\text { Reforçamento Positivo }\end{array}$ & 3 \\
\hline $\begin{array}{l}\text { 2. Jerome e } \\
\text { Sturmey(2008) }\end{array}$ & $\begin{array}{l}\text { Reinforcing Efficacy } \\
\text { of Interactions } \\
\text { with Preferred and } \\
\text { Nonpreferred Staff }\end{array}$ & $\begin{array}{l}\text { Queens College and } \\
\text { The Graduate Center } \\
\text { City University Of } \\
\text { New York }\end{array}$ & $\begin{array}{l}\text { Comparação de ava- } \\
\text { liações de preferência } \\
\text { por figura ou verbal }\end{array}$ & $\begin{array}{l}\text { Avaliação de prefe- } \\
\text { rência } \\
\text { Instrução } \\
\text { Reforçamento positivo }\end{array}$ & 2 \\
\hline $\begin{array}{l}\text { 3. Athens, } \\
\text { Vollmer,Sloman } \\
\text { e Pipkin(2008) }\end{array}$ & $\begin{array}{l}\text { An Analysis of Vocal } \\
\text { Stereotypy and } \\
\text { Therapist Fading }\end{array}$ & $\begin{array}{l}\text { University of Florida } \\
\text { West Virginia } \\
\text { University }\end{array}$ & $\begin{array}{l}\text { Reduzir estereotipia } \\
\text { vocal }\end{array}$ & $\begin{array}{l}\text { Análise funcional } \\
\text { Reforçamento positivo } \\
\text { Modelação } \\
\text { Fading }\end{array}$ & 1 \\
\hline
\end{tabular}




\begin{tabular}{|c|c|c|c|c|c|}
\hline $\begin{array}{l}\text { 4. Taylor e } \\
\text { Hoch(2008) }\end{array}$ & $\begin{array}{l}\text { Teaching Children } \\
\text { with Autism to } \\
\text { Respond to and } \\
\text { Initiate Bids for Joint } \\
\text { Attention }\end{array}$ & Alpine Learning Group & $\begin{array}{l}\text { Iniciar atenção com- } \\
\text { partilhada }\end{array}$ & $\begin{array}{l}\text { Atraso de dica } \\
\text { Prompting } \\
\text { Reforçamento positivo }\end{array}$ & 3 \\
\hline $\begin{array}{l}\text { 5. Tarbox et } \\
\text { al.(2009) }\end{array}$ & $\begin{array}{l}\text { Use of Chaining to } \\
\text { Increase Complexity } \\
\text { of Echoics in } \\
\text { Children with Autism }\end{array}$ & $\begin{array}{l}\text { Center for Autism and } \\
\text { Related Disorders }\end{array}$ & $\begin{array}{l}\text { Aumentar repertório } \\
\text { de ecóicos }\end{array}$ & $\begin{array}{l}\text { Encadeamento } \\
\text { Modelação } \\
\text { Reforçamento positivo }\end{array}$ & 3 \\
\hline $\begin{array}{l}\text { 6. Macdonald et } \\
\text { al. (2009) }\end{array}$ & $\begin{array}{l}\text { Using Video } \\
\text { Modeling to Teach } \\
\text { Reciprocal Pretend } \\
\text { pPay to Children } \\
\text { with Autism }\end{array}$ & $\begin{array}{l}\text { New England Center } \\
\text { for Children } \\
\text { Northeastern } \\
\text { University }\end{array}$ & $\begin{array}{l}\text { Ensinar brincadeira } \\
\text { recíproca }\end{array}$ & $\begin{array}{l}\text { Instrução } \\
\text { Modelação } \\
\text { Prompting } \\
\text { Reforçamento positivo }\end{array}$ & 4 \\
\hline $\begin{array}{l}\text { 7. Esch, Carr e } \\
\text { Grow (2009) }\end{array}$ & $\begin{array}{l}\text { Evaluation of } \\
\text { an Enhanced } \\
\text { Stimulus-Stimulus } \\
\text { Pairing Procedure } \\
\text { to Increase Early } \\
\text { Vocalizations of } \\
\text { Children with Autism }\end{array}$ & $\begin{array}{l}\text { Western Michigan } \\
\text { University }\end{array}$ & $\begin{array}{l}\text { Aumentar o repertório } \\
\text { inicial de vocalizações }\end{array}$ & $\begin{array}{l}\text { Pareamento de estímu- } \\
\text { los } \\
\text { Prompting } \\
\text { Instruções }\end{array}$ & 3 \\
\hline $\begin{array}{l}\text { 8. Murphy } \\
\text { e Barnes- } \\
\text { Holmes(2009) }\end{array}$ & $\begin{array}{l}\text { Derived More-Less } \\
\text { Relational Mands in } \\
\text { Children Diagnosed } \\
\text { with Autism }\end{array}$ & $\begin{array}{l}\text { National University of } \\
\text { Ireland }\end{array}$ & Relações derivadas & $\begin{array}{l}\text { Emparelhamento com } \\
\text { o modelo } \\
\text { Reforçamento positivo }\end{array}$ & 4 \\
\hline $\begin{array}{l}\text { 9. Marcus e } \\
\text { Wilder (2009) }\end{array}$ & $\begin{array}{l}\text { A Comparison of } \\
\text { Peer Video Modeling } \\
\text { and Self-Video } \\
\text { Modeling to Teach } \\
\text { Textual Responses } \\
\text { in Children with } \\
\text { Autism }\end{array}$ & $\begin{array}{l}\text { Florida Institute of } \\
\text { Technology }\end{array}$ & $\begin{array}{l}\text { Ensino de respostas } \\
\text { textuais }\end{array}$ & $\begin{array}{l}\text { Reforçamento positivo } \\
\text { Modelação }\end{array}$ & 3 \\
\hline $\begin{array}{l}\text { 10. Reagon, e } \\
\text { Higbee(2009) }\end{array}$ & $\begin{array}{l}\text { Parent-Implemented } \\
\text { Script Fading to } \\
\text { Promote Play Based } \\
\text { Verbal Initiations in } \\
\text { Children with Autism }\end{array}$ & Utah State University & $\begin{array}{l}\text { Pais ajudarem na } \\
\text { promoção de verbali- } \\
\text { zações iniciais }\end{array}$ & $\begin{array}{l}\text { Instrução } \\
\text { Prompting } \\
\text { Fading } \\
\text { Reforçamento positivo }\end{array}$ & 3 \\
\hline $\begin{array}{l}\text { 11. Shillingsburg } \\
\text { et al.(2009) }\end{array}$ & $\begin{array}{l}\text { Evaluation and } \\
\text { Training of Yes-No } \\
\text { Responding Across } \\
\text { Verbal Operants }\end{array}$ & $\begin{array}{l}\text { Marcus Institute and } \\
\text { Emory University } \\
\text { School of Medicine } \\
\text { University of } \\
\text { Southern Maine and } \\
\text { Providence's Achieve } \\
\text { Munroe-Meyer } \\
\text { Institute and University } \\
\text { of Nebraska } \\
\text { Medical Center }\end{array}$ & $\begin{array}{l}\text { Independência fun- } \\
\text { cional dos operantes } \\
\text { verbais }\end{array}$ & $\begin{array}{l}\text { Avaliação de prefe- } \\
\text { rencia } \\
\text { Reforçamento positivo } \\
\text { Prompting }\end{array}$ & 3 \\
\hline $\begin{array}{l}\text { 12. Kodak e } \\
\text { Clements(2009) }\end{array}$ & $\begin{array}{l}\text { Acquisition of } \\
\text { Mands and Tacts } \\
\text { with Concurrent } \\
\text { Echoic Training }\end{array}$ & $\begin{array}{l}\text { Munroe-Meyer } \\
\text { Institute and University } \\
\text { of Nebraska Medical } \\
\text { Center }\end{array}$ & $\begin{array}{l}\text { Aquisição de mando e } \\
\text { de tato }\end{array}$ & $\begin{array}{l}\text { Reforçamento positivo } \\
\text { Prompting } \\
\text { Instrução }\end{array}$ & 1 \\
\hline $\begin{array}{l}\text { 13. Ingvarsson } \\
\text { e Hollobaugh } \\
\text { (2010) }\end{array}$ & $\begin{array}{l}\text { Acquisition of } \\
\text { Intraverbal Behavior: } \\
\text { Teaching Children } \\
\text { with Autism to Mand } \\
\text { for Answers to } \\
\text { Questions }\end{array}$ & $\begin{array}{l}\text { University of North } \\
\text { Texas } \\
\text { Child Study Center, } \\
\text { Fort Worth } \\
\text { Youngstown State } \\
\text { University }\end{array}$ & $\begin{array}{l}\text { Aquisição de intra- } \\
\text { verbal }\end{array}$ & $\begin{array}{l}\text { Prompting } \\
\text { Reforçamento positivo }\end{array}$ & 3 \\
\hline
\end{tabular}




\begin{tabular}{|c|c|c|c|c|c|}
\hline $\begin{array}{l}\text { 14. Murphy } \\
\text { e Barnes- } \\
\text { Holmes(2010) }\end{array}$ & $\begin{array}{l}\text { Establishing Five } \\
\text { Derived Mands in } \\
\text { Three Adolescent } \\
\text { Boys with Autism }\end{array}$ & $\begin{array}{l}\text { National University of } \\
\text { Ireland }\end{array}$ & $\begin{array}{l}\text { Relações derivadas de } \\
\text { mandos }\end{array}$ & $\begin{array}{l}\text { Reforçamento positivo } \\
\text { Emparelhamento com } \\
\text { o modelo }\end{array}$ & 3 \\
\hline $\begin{array}{l}\text { 15. Carbone et } \\
\text { al.(2010) }\end{array}$ & $\begin{array}{l}\text { Increasing the } \\
\text { Vocal Responses of } \\
\text { Children with Autism } \\
\text { and Developmental } \\
\text { Disabilities using } \\
\text { Manual Sign Mand } \\
\text { Training and Prompt } \\
\text { Delay }\end{array}$ & $\begin{array}{l}\text { Carbone Clinic } \\
\text { Verbal Behavior } \\
\text { Institute } \\
\text { Center for Autism } \\
\text { Treatment }\end{array}$ & $\begin{array}{l}\text { Aumento de respostas } \\
\text { vocais iniciais }\end{array}$ & $\begin{array}{l}\text { Atraso de dica } \\
\text { Reforçamento positivo }\end{array}$ & 3 \\
\hline $\begin{array}{l}\text { 16. Plavnick e } \\
\text { Ferreri }(2011)\end{array}$ & $\begin{array}{l}\text { Establishing Verbal } \\
\text { Repertoires in } \\
\text { Children with Autism } \\
\text { Using Function- } \\
\text { Based Video } \\
\text { Modeling }\end{array}$ & $\begin{array}{l}\text { Michigan State } \\
\text { University }\end{array}$ & $\begin{array}{l}\text { Ensino de repertório } \\
\text { verbal (mandos) }\end{array}$ & $\begin{array}{l}\text { Análise funcional } \\
\text { Avaliação de prefe- } \\
\text { rência } \\
\text { Reforçamento positivo } \\
\text { Modelação }\end{array}$ & 4 \\
\hline $\begin{array}{l}\text { 17. Betz et } \\
\text { al.(2011) }\end{array}$ & $\begin{array}{l}\text { Increasing } \\
\text { Response Variability } \\
\text { of Mand Frames } \\
\text { with Script Training } \\
\text { and Extinction. }\end{array}$ & Utah State University & $\begin{array}{l}\text { Variabilidade de res- } \\
\text { postas de mando }\end{array}$ & $\begin{array}{l}\text { Instrução } \\
\text { Prompting } \\
\text { Fading } \\
\text { Reforçamento positivo }\end{array}$ & 3 \\
\hline $\begin{array}{l}\text { 18. Ingvarsson } \\
\text { e Hollobaugh } \\
\text { (2011) }\end{array}$ & $\begin{array}{l}\text { An Comparing } \\
\text { of Prompting } \\
\text { Tactics to Establish } \\
\text { Intraverbals in } \\
\text { Children with Autism }\end{array}$ & $\begin{array}{l}\text { University Of North } \\
\text { Texas } \\
\text { Child Study Center, } \\
\text { Fort Worth } \\
\text { Youngstown State } \\
\text { University }\end{array}$ & Ensino de intraverbais & $\begin{array}{l}\text { Prompting } \\
\text { Reforçamento positivo }\end{array}$ & 3 \\
\hline $\begin{array}{l}\text { 19. Pollard, Betz } \\
\text { e Higbee (2012) }\end{array}$ & $\begin{array}{l}\text { Script Fading to } \\
\text { Promote Unscripted } \\
\text { Bids for Joint } \\
\text { Attention in Children } \\
\text { with Autism }\end{array}$ & Utah State University & $\begin{array}{l}\text { Iniciar atenção com- } \\
\text { partilhada }\end{array}$ & $\begin{array}{l}\text { Instrução } \\
\text { Prompting } \\
\text { Fading } \\
\text { Reforçamento positivo }\end{array}$ & 3 \\
\hline $\begin{array}{l}\text { 20. Colon, } \\
\text { Ahearn, Clark e } \\
\text { Masalsky(2012) }\end{array}$ & $\begin{array}{l}\text { The Effects of Verbal } \\
\text { Operant Training } \\
\text { and Response } \\
\text { Interruption and } \\
\text { Redirection on } \\
\text { Appropriate and } \\
\text { Inappropriate } \\
\text { Vocalizations }\end{array}$ & $\begin{array}{l}\text { New England Center } \\
\text { for Children } \\
\text { Northeastern } \\
\text { University }\end{array}$ & $\begin{array}{l}\text { Redução de estereoti- } \\
\text { pia verbal }\end{array}$ & $\begin{array}{l}\text { Bloqueio de resposta } \\
\text { Interrupção de res- } \\
\text { posta } \\
\text { Avaliação de prefe- } \\
\text { rência } \\
\text { Análise funcional } \\
\text { Reforçamento positivo } \\
\text { Prompting }\end{array}$ & 3 \\
\hline $\begin{array}{l}\text { 21. Marchese et } \\
\text { al.(2012) }\end{array}$ & $\begin{array}{l}\text { The Effects of the } \\
\text { Question "What is } \\
\text { This?" On Tact- } \\
\text { Training Outcomes } \\
\text { of Children with } \\
\text { Autism }\end{array}$ & $\begin{array}{l}\text { Kinark Child and } \\
\text { Family Services } \\
\text { Auburn University }\end{array}$ & $\begin{array}{l}\text { Ensino de tatos com } \\
\text { ou sem SD }\end{array}$ & $\begin{array}{l}\text { Prompting } \\
\text { Reforçamento positivo }\end{array}$ & 4 \\
\hline $\begin{array}{l}\text { 22. Finn, Miguel } \\
\text { e Ahearn (2012) }\end{array}$ & $\begin{array}{l}\text { The Emergence of } \\
\text { Untrained Mands } \\
\text { and Tacts in } \\
\text { Children with Autism }\end{array}$ & $\begin{array}{l}\text { New England } \\
\text { Center for Children } \\
\text { and Northeastern } \\
\text { University } \\
\text { California State } \\
\text { University }\end{array}$ & $\begin{array}{l}\text { Emergência de } \\
\text { operantes verbais } \\
\text { não treinados (tatos e } \\
\text { mandos) }\end{array}$ & $\begin{array}{l}\text { Encadeamento } \\
\text { Prompting } \\
\text { Reforçamento positivo }\end{array}$ & 4 \\
\hline $\begin{array}{l}\text { 23. Polick, Carr } \\
\text { e Hanney (2012) }\end{array}$ & $\begin{array}{l}\text { A Comparison } \\
\text { of General and } \\
\text { Descriptive Praise in } \\
\text { Teaching Intraverbal } \\
\text { Behavior to Children } \\
\text { with Autism }\end{array}$ & $\begin{array}{l}\text { Florida State } \\
\text { University } \\
\text { Auburn University }\end{array}$ & $\begin{array}{l}\text { Aquisição e manuten- } \\
\text { ção de intraverbal }\end{array}$ & Reforçamento positivo & 2 \\
\hline
\end{tabular}




\begin{tabular}{|c|c|c|c|c|c|}
\hline $\begin{array}{l}\text { 24. Valentino, } \\
\text { Shillingsbu e } \\
\text { Call(2012) }\end{array}$ & $\begin{array}{l}\text { Comparing the } \\
\text { Effects of Echoic } \\
\text { Prompts and Echoic } \\
\text { Prompts plus } \\
\text { Modeled Prompts } \\
\text { on Intraverbal } \\
\text { Behavior }\end{array}$ & $\begin{array}{l}\text { Marcus Autism Center } \\
\text { and Emory University }\end{array}$ & $\begin{array}{l}\text { Ensinar respostas } \\
\text { verbais de intraverbal }\end{array}$ & $\begin{array}{l}\text { Prompting } \\
\text { Reforçamento positivo }\end{array}$ & 1 \\
\hline $\begin{array}{l}\text { 25. Grannan e } \\
\text { Rehfeldt (2012) }\end{array}$ & $\begin{array}{l}\text { Emergent Intraverbal } \\
\text { Responses via Tact } \\
\text { andMatch-to-Sample } \\
\text { Instruction }\end{array}$ & $\begin{array}{l}\text { Southern Illinois } \\
\text { University }\end{array}$ & $\begin{array}{l}\text { Emergência de intra- } \\
\text { verbal }\end{array}$ & $\begin{array}{l}\text { Emparelhamento com } \\
\text { o modelo } \\
\text { Reforçamento positivo } \\
\text { Prompting }\end{array}$ & 2 \\
\hline $\begin{array}{l}\text { 26. Bowen, } \\
\text { Shillingsburg e } \\
\text { Carr (2012) }\end{array}$ & $\begin{array}{l}\text { The Effects of the } \\
\text { Question "What } \\
\text { do You Want?" } \\
\text { On Mand Training } \\
\text { Outcomes of } \\
\text { Children with Autism }\end{array}$ & $\begin{array}{l}\text { Marcus Autism Center } \\
\text { Emory University } \\
\text { School of Medicine } \\
\text { Auburn University }\end{array}$ & $\begin{array}{l}\text { Treino de mando com } \\
\text { e sem dica verbal }\end{array}$ & $\begin{array}{l}\text { Avaliação de prefe- } \\
\text { rencia } \\
\text { Prompting } \\
\text { Atraso de dica } \\
\text { Reforçamento positivo }\end{array}$ & 2 \\
\hline $\begin{array}{l}\text { 27. Marion et al. } \\
(2012)\end{array}$ & $\begin{array}{l}\text { Teaching Children } \\
\text { with Autism } \\
\text { Spectrum Disorder } \\
\text { to Mand for } \\
\text { Information Using } \\
\text { "Which?" }\end{array}$ & $\begin{array}{l}\text { University of Manitoba } \\
\text { St. Amant Research } \\
\text { Centre }\end{array}$ & Ensinar mando & $\begin{array}{l}\text { Avaliação de prefe- } \\
\text { rência } \\
\text { Prompting } \\
\text { Reforçamento } \\
\text { Operações estabele- } \\
\text { cedoras forçadas }\end{array}$ & 3 \\
\hline
\end{tabular}

Em relação à distribuição das publicações no período citado, verificamos que os anos de 2009 e de 2012 tiveram o maior número de publicações, oito e nove artigos, respectivamente. Em 2010 e 2011, foram publicados três artigos em cada ano e, em 2008, quatro artigos. Não foi possível analisar de maneira qualitativa os dados pois o período compreendido nesta revisão não permitiu uma comparação mais extensa entre os anos e as décadas de existência do periódico (Tabela 1).

O tema de estudo de cada artigo é apresentado na Tabela 2 e foi elaborado a partir dos objetivos e títulos descritos em cada estudo. Em linhas gerais, grande parte dos artigos se dedicou ao ensino de novos repertórios e apenas dois voltaram-se para a redução de excessos comportamentais - nesse caso, estereotipias vocais. Observamos ainda que, em sua grande maioria, os estudos se dedicaram a ensinar a linguagem a partir da taxonomia descrita por Skinner (1957), com destaque especial para o ensino e/ou emergência de mandos. Além disso, a revisão mostrou estudos com interesse em ensi- nar os participantes a iniciar uma interação social (e.g., iniciar verbalizações, atenção compartilhada e brincar compartilhado). Frequentemente, mais de um operante verbal esteve envolvido em cada estudo.

Para Plavnick e Ferreri (2011), intervenções que ensinam o indivíduo a comunicar-se em uma variedade de situações estão entre as práticas essenciais para indivíduos autistas, sendo o treino de mandos um dos primeiros passos em qualquer programa de ensino para habilidades comunicativas. Os estudos recentes encontrados nesta revisão confirmam essa preocupação da área.

A capacidade de pedir e de recusar coisas de modo funcional é fundamental para a redução de comportamentos inadequados, tais como birras e agressões (Carbone et al., 2010). Além disso, o ensino de mando possibilita a aquisição de outras competências linguísticas como o tato (Egan \& Barnes-Holmes, 2009) e ainda permite o ensino concomitante de repertório ecóico (Kodak \& Clement, 2009). Finalmente, o ensino do mando 
possibilita que a criança desenvolva outras habilidades sociais, tais como iniciar interações e brincar recíproco (Pollard, Betz \& Higbee,2012).

Tabela 2 - Tema de Estudo dos Artigos Avaliados na Presente Revisão de Literatura

\begin{tabular}{lll}
\hline Tema & & $\mathbf{N}^{\circ}$ de artigos \\
\hline $\begin{array}{l}\text { Iniciação de verbalização } \\
\text { Iniciar atenção }\end{array}$ & 1 \\
compartilhada & 2 \\
$\begin{array}{l}\text { Brincar compartilhado } \\
\text { Ensino e/ou emergência }\end{array}$ & Ecóico & 3 \\
de novos operantes & Mando & 11 \\
& Tato & 4 \\
& Intraverbal & 6 \\
& Textual & 1 \\
Preferência/escolha & & 1 \\
Redução de estereotipia vocal & 2
\end{tabular}

Além de saber pedir, descrever os vários aspectos do ambiente interno e externo é essencial para que a linguagem se desenvolva. Um grande repertório de tatos é instalado nos falantes desde a infância, como quando a criança, por exemplo na presença da mãe, recebe um afago ou sorriso ao falar a palavra mãe. $\mathrm{O}$ ensino de tato deve estar presente o mais cedo possível nos programas curriculares das crianças autistas (Marchese et al., 2012), pois, como já referido, o interesse diminuído por situações mediadas por outras pessoas não favorece a aquisição desse repertório.

Entre os resultados desta revisão, encontramos também o ensino dos intraverbais. $\mathrm{O}$ comportamento intraverbal desempenha papel importante nas interações sociais (e.g., conversas, canções e descrição de uma história) sendo, em parte, produto de processos de ensino que reconhecemos como habilidades acadêmicas. Destacamos o ensino de tato para emergência de respostas intraverbais (Grannan \& Rehfeldt, 2012), bem como o uso de ecóicos (Valentino \& Shillingsburg, 2012) apresentados na Tabela 2 .

Uma variedade de procedimentos foi adotada, como mostra a Figura 1. Evidenciamos o uso do reforçamento positivo (e.g., elogios, elogios descritivos, comestíveis, fichas, atividades, objetos e atenção) e os procedimentos relacionados ao controle de estímulo (i.e., dicas - prompting, modelação, encadeamento, fading) porque foram utilizados em praticamente todos os estudos em algum momento do procedimento. Os procedimentos de emparelhamento com o modelo (MTS), pela técnica específica de execução, foram considerados como uma unidade de análise e encontrados em três dos estudos. Avaliações de preferência (AP) foram realizadas em seis estudos e análise funcional (AF) em três estudos. Todos os outros

\section{Figura 1 - Procedimentos adotados no desenvolvimento das pesquisas} revistas no presente estudo.

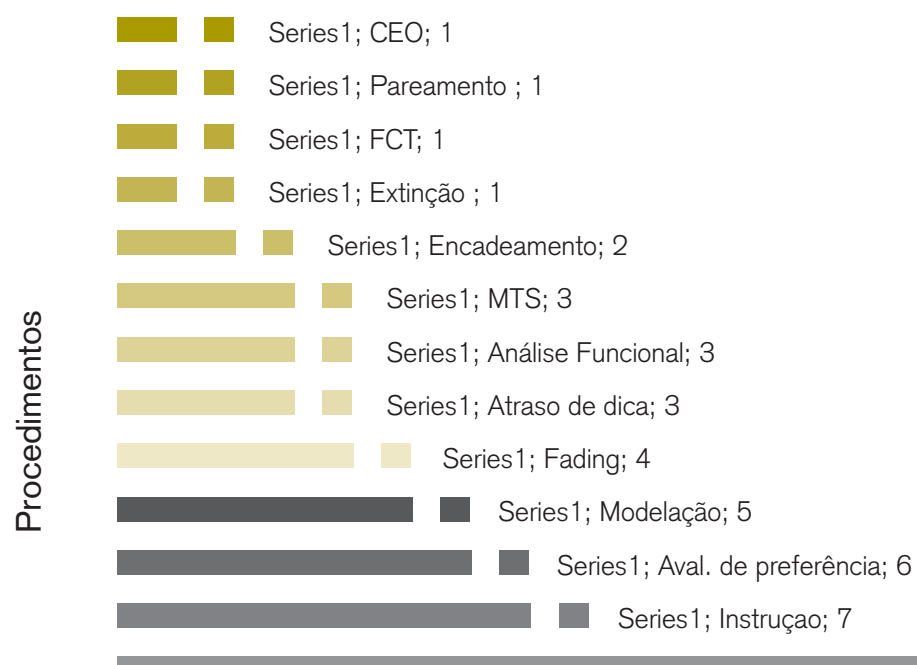


procedimentos apresentados, incluindo o treino de comunicação funcional (FCT) e o de operação estabelecedora forçada (CEO), tiveram uma ocorrência. Lembramos ainda que, em quase todos os artigos, diferentes procedimentos foram combinados.

Corroborando os achados deste estudo, Nicolino e Zanotto (2010) apontaram que, para a educação especial, os procedimentos relacionados ao controle de estímulos foram os mais frequentes entre os anos de 2001 a 2008 no JABA. Da mesma forma, Petursdottir e Carr (2011) ressaltaram os procedimentos de reforçamento, reforçamento diferencial e controle de estímulos como os mais usuais para pessoas com transtornos do desenvolvimento.

Finalmente, as discussões referentes aos estudos futuros destacaram a relevância da generalização dos comportamentos aprendidos. Athens, Vollmer, Sloman e Pipkin (2008) discutem, por exemplo, a importância de envolver os pais no treinamento. Além disso, o ensino em ambiente natural (e.g., escolas, parques e eventos cotidianos) é apontado por MacDonald et al. (2009) e Taylor e Hock (2008) como o principal fator para que os novos comportamentos sejam mantidos, além de indicar uma opção de ensino combinada, com procedimentos de tentativa discreta e ensino em ambiente natural. Este último permite a continuidade do ensino da linguagem na presença dos estímulos e variáveis motivacionais que deverão, eventualmente, controlar e manter o comportamento verbal da criança.

Outro tópico destacado para estudos futuros é que seja realizado uma descrição mais apurada do repertório verbal inicial dos participantes. Segundo Petursdottir e Carr (2011), os resultados obtidos algumas vezes garantem que a manipulação das variáveis de cada estudo foi responsável pela aquisição do novo repertório verbal.

\section{Considerações Finais}

Estima-se que os custos pessoais, sociais e financeiros oriundos das necessidades daqueles que convivem com indivíduos diagnosticados com autismo sejam bastante elevados. Nessa conta, pode-se incluir os custos com o tratamento, tanto na área da saúde quanto escolar, bem como a perda de produtividade pessoal ou de membros da família (Bouder, Spielman \& Mandell, 2009). A implementação de intervenções precoces, psicoeducacionais e prolongadas propicia melhor prognóstico, bem como redução dos custos financeiros e sociais. Nesta revisão, pudemos constatar que a análise do comportamento tem contribuído de forma persistente no desenvolvimento de recursos que melhoram a condição dessa população, sobretudo ajudando na produção de tecnologia para o ensino da linguagem. Os limites deste estudo se devem ao período restrito de tempo selecionado e à escolha de somente um periódico. Futuros estudos poderão realizar revisões em um período de tempo mais extenso, bem comoincluir outros periódicos da área, inclusive periódicos nacionais dedicados a publicações referentes à educação especial - o que certamente contribuirá para as lacunas e enriquecimento desse estudo.

\section{Referências}

American Psychiatric Association, [APA]. (2003). Diagnostic and Statistical Manual of Mental Disorders (4a ed. rev.). Washington: Autor.

American Psychiatric Association, [APA]. (2013). Diagnostic and Statistical Manual of Mental Disorders (5a ed.). Washington: Autor.

Athens, E. S., Vollmer, T. R., Sloman, K. N., \& Pipkin, C. S. P. (2008). An analysis of vocal stereotypy and therapist fading. Journal of Applied Behavior Analysis, 41, 291-297.

Barbera, M. L. (2007). The verbal behavior approach: How to teach children with autism and related disorders. London: Jessica Kingsley.

Baron-Cohen, S. (2000). Theory of mind and autism: A fifteen year review. Em S. Baron-Cohen, H. Tager-Flusberg \& D. J. Cohen (Orgs.), Understanding other minds: Perspectives from developmental cognitive neuroscience (2a ed.; pp. 3-20). New York: Oxford University Press.

Belisário Filho, J. F., \& Cunha, P. (2010). A educação especial na perspectiva da inclusão escolar: Transtornos globais do desenvolvimento. Brasília: Ministério da Educação, Secretaria de Educação Especial.

Betz, A. M., Higbee, T. S, Kelley, K. N., Sellers, T. P., \& Pollard, J. S. (2011). Increasing response variability of mand frames with script training and extinction. Journal of Applied Behavior Analysis, 44, 357-362. 
Bosa, C. A. (2001). As relações entre autismo, comportamento social e função executiva. Psicologia: Reflexão e Crítica, 14, 281-287.

Bouder, J. N., Spielman, S., \& Mandell, D. S. (2009). Quantifying the impact of autism coverage on private insurance premiums. Journal of Autism and Developmental Disorder, 39, 953-957.

Bowen, C. N., Shillingsburg, M. A., \& Carr, J. E. (2012).The effects of the question "What do you want?" On mand training outcomes of children with autism. Journal of Applied Behavior Analysis, 45,833-838.

Carbone, V. J., Sweeney-Kerwin, E. J., Attanasio, V., \& Kasper, T. (2010). Increasing the vocal responses of children with autism and developmental disabilities using manual sign mand training and prompt delay. Journal of Applied Behavior Analysis, 43, 705-709.

Carr, J. E., \& Firth, A. M. (2005). The verbal behavior approach to early and intensive behavioral intervention for autism: A call for additional empirical support. Journal of Early and Intensive Behavior Intervention, 2, 18-27.

Colon, C. L., Ahearn, W. H., Clark, K. M., \& Masalsky, J. (2012).The effects of verbal operant training and response interruption and redirection on appropriate and inappropriate vocalizations. Journal of Applied Behavior Analysis, 45, 107-120.

Finn, H. E., Miguel, C. F., \& Ahearn, W. H. (2012). The emergence of untrained mands and tacts in children with autism. Journal of Applied Behavior Analysis, 45, 265-280.

Fombonne, E. (2009). Epidemiology of pervasive developmental disorders. Pediatric Reserch, 65, 591-598.

Grannan, L., \& Rehfeldt, R. A. (2012). Emergent intraverbal responses via tact and match-to-sample instruction. Journal of Applied Behavior Analysis, 45, 601-605.

Greer, R. D., \& Ross, D. E. (2008). Verbal behavior analysis: Inducing and expanding complex communication in children with severe language delays. Boston: Allyn \& Bacon.

Grow, L. L., Kelley, M. E., Roane, H. S., \& Shillingsburg, M. A. (2008). Utility of extinction-Induced response variability for the selection of mands. Journal of Applied Behavior
Analysis, 41, 15-24.

Ingvarsson, E. T., \& Hollobaugh, T. (2010). Acquisition of intraverbal behavior: Teaching children with autism mand for answers to questions. Journal of Applied Behavior Analysis, 43, $1-17$.

Ingvarsson, E. T., \& Hollobaugh, T. (2011). A comparison of prompting tactics to establish intraverbal in clildren with autism. Journal of Applied Behavior Analysis, 44, 659-664.

Jerome, J., \& Sturmey, P. (2008). Reinforcing efficacy of interactions with preferred and non- preferred staff under progressive-ratio schedules. Journal of Applied Behavior Analysis, 41, 221-225.

Kodak, T., \& Clements, A. (2009). Acquisition of mands and tacts with concurrent echoic training. Journal of Applied Behavior Analysis, 42, 839-843.

Lampreia, C. (2007). A perspectiva desenvolvimentista para a intervenção precoce no autismo. Estudos de Psicologia, 24, 105-114.

Leaf, R., McEachin, J., \& Harsh, J. D. (1999). A work in progress: Behavior management strategies and a curriculum for intensive behavioral treatment of autism. New York: DRL.

LeBlanc, L., \& Gillis, J. M. (2012). Behavioral interventions for children with autism spectrum disorders. Pediatric Clinical, 59, 147-164.

Lovaas, O. I. (1987). Behavioral treatment and normal educational and intellectual functioning in young autistic children. Journal of Consulting and Clinical Psychology, 55, 3-9.

Marchese, N. V., Carr, J. E., Leblanc, L. A., Rosati, T. C., \& Conroy, A. A. (2012). The effects of the question "What is this?" on tact-training outcomes of children with autism. Journal of Applied Behavior Analysis, 45, 539-547.

Marcus, A., \& Wilder, D.A. (2009). A comparison of peer video modeling and self-video modeling to teach textual responses in children with autism. Journal of Applied Behavior Analysis, 42, 335-341.

Macdonald, R., Sacramone, S., Mansfield, R., Wiltz, K., \& Ahearn, W. H. (2009). Using video modeling to teach reciprocal pretend play to children with autism. Journal of Applied Behavior Analysis, 42, 43-55. 
Marion, C., Martin, G. L., Yu, C. T., Buhler, C., Kerr, D., \& Claeys, A. (2012). Teaching children with autism spectrum disorder to mand for information using "which?". Journal of Applied Behavior Analysis, 45, 865-870.

Maurice, C. (1993). Let me hear your voice: A family's triumph over autism. New York: Ballantine Books.

Maurice, C., Green, G., \& Luce, S. (1996). Behavioral intervention for young children with autism: A manual for parents and professionals. Austin: Pro-Ed.

Murphy, C., \& Barnes-Holmes, D. (2009). Derived more-less relation mands in children with autism. Journal of Applied Behavior Analysis, 42, 253-268.

Murphy, C., \& Barnes-Holmes, D. (2010). Establishing five derived mands in three adolescent boys with autism. Journal of Applied Behavior Analysis, 43, 537-541.

Newschaffer, C. J., Croen, L. A., Daniels, J., Giarelli, E., Grether, J. K., Levy, S. E., . . . Windham, G. C. (2007). The epidemiology of autism spectrum disorders. Annual Review of Public Health 28, 235-258.

Nicolino, V. F., \& Zanoto, M. L. B. (2010). Revisão histórica de pesquisas em Análise do Comportamento e educação especial/inclusão publicadas no JABA entre 2001 e 2008. Psicologia: Teoria e Prática, 12, 51-79.

Nunes, D. R. P., \& Nunes Sobrinhos, F. P. (2010). Comunicação alternativa e ampliada para educandos com autismo: Considerações metodológicas. Revista Brasileira de Educação Especial, 16, 297-312.

Partington, J. W., \& Sundberg, M. L. (1998). The assessment of basic language and learning skills: The ABLLS. Pleasant Hill: Behavior Analysts.

Paula, C. S.,Ribeiro, S., Fombonne, E., \& Mercadante, M. T. (2011). Prevalence of pervasive developmental disorder in Brazil: A pilot study. Journal of Autism and Developmental Disorders, 41, 1738-1742.

Petursdottir, A. I., \& Carr, J. E. (2011). A review of recommendations for sequencing receptive and expressive language instruction. Journal of Applied Behavior Analysis, 44, 859-876.
Plavnick, J. B., \& Ferreri, S. J. (2011). Establishing verbal repertories in children with autismo using function-based video modeling. Journal of Applied Behavior Analysis, 44, 747-766.

Pollard, J. S., Betz, A. M., \& Higbee, T. S. (2012). Script fading to promote unscripted bids for joint attention in children with autism. Journal of Applied Behavior Analysis, 45, 387-393.

Polick, A. S., Carr, J. E., \& Hanney, N. M. (2012). A comparison of general and descriptive praise in teaching intraverbal to children with autism. Journal of Applied Behavior Analysis, 45, 593599.

Rapin, I. (1991). Autistic children: Diagnosis and clinical features. Pediatrics, 87,751-760.

Reagon, K. A., \& Higbee, T. S. (2009). Parentimplemented script fading to promote play-based verbal initiations in children with autism. Journal of Applied Behavior Analysis, 42, 659664.

Reichow, B., \& Volkmar, F. R. (2010). Social skills interventions for individuals with autism: Evaluation for evidence-based practices within a best evidence synthesis framework. Journal of Autism and Developmental Disorders, 40, 149166.

Rice, C. (2009). Autism and developmental disabilities monitoring network, United States, 2006. Morbidity and Mortality Weekly ReportMMWR,58, 1-20.

Secretaria dos Direitos da Pessoa com Deficiência. (2013). Protocolo do estado de São Paulo de diagnóstico, tratamento e encaminhamento de pacientes com transtorno do espectro autista (TEA). Governo do Estado de São Paulo. Retirado de http://www.pessoacomdeficiencia. sp.gov.br/usr/share/documents/ProtocoloEstadodeSaoPaulodeDiagnosticoTEA.pdf

Shillingsburg, M. A., Kelley, M. E., Roane, H. S., Kisamor, A., \& Brown, M. R. (2009). Evaluation and training of yes-no responding across verbal operants. Journal of Applied Behavior Analysis, 42, 209-223.

Skinner, B. F. (1957). Verbal behavior. New York: Appleton-Century-Crofts.

Sundberg, M. L. (2008). The verbal behavior milestones assessment and placement program: The VB-MAPP. Concord: AVB Press. 
Suplino, M. H. F. O. (2007). Retratos e imagens das vivências inclusivas de dois alunos com autismo em classes regulares. (Tese de Doutorado). Universidade Estadual do Rio de Janeiro, Rio de Janeiro, RJ.

Tarbox, J., Madrid, W., Aguilar, B., Jacobo, W., \& Schiff, A. (2009). Use of chaining to increase complexity of echoic in children with autism. Journal of Applied Behavior Analysis, 42, 901906.

Taylor, B. A., \& Hoch, H. (2008). Teaching children with autism to respond to and initiate bids for joint attention. Journal of Applied Behavior Analysis, 41, 377-391.

Valentino, A. L., Shillingsburg, M. A., \& Call, N. A. (2012). Comparing the effects of echoic prompts and echoic prompts plus modeled prompts on intraverbal behavior. Journal of Applied Behavior Analysis, 45, 431-435.

Velasco, S. M., Mijares, M. G, \& Tomanari, G. Y. (2010). Fundamentos metodológicos da pesquisa em análise experimental do comportamento. Psicologia em Pesquisa, 4,150-155.

Virués-Ortega, J. (2010). Applied behavior analytic intervention for autism in early childhood: Meta-analysis, meta regression and dose-response meta-analysis of multiple outcomes. Clinical Psychology Review, 30, 387-399.

Werner, E., Dawson, G., Munson, J., \& Osterling, J. (2005). Variation in early developmental course in autism and its relation with behavioral outcome at 3-4 years of age. Journal of Autism and Developmental Disorder,35, 337-350

\section{Informações do Artigo}

Histórico do artigo:

Submetido em: 20/04/2013

Primeira decisão editorial: 16/06/2013

Segunda decisão editorial: 24/06/2013

Aceito em: 02/07/2013 